\title{
Cuidado perdido de enfermería y su relación con el síndrome de agotamiento profesional
}

\author{
Nursing missed care and its relationship \\ with burnout syndrome
}

\section{Cuidado perdido de enfermagem e sua relação com a síndrome de esgotamento profissional}

\author{
M.F. Rabadán-Silva ${ }^{a^{*}}$, R.A. Zárate-Grajales ${ }^{\mathrm{b} 2}$, \\ L.Á. Benítez-Chavira ${ }^{\mathrm{c} 2}$ \\ ORCID: \\ ${ }^{\mathrm{a}} \underline{0000-0002-3484-9585} \quad{ }^{\mathrm{b}} \underline{0000-0002-9264-8490} \quad{ }^{\mathrm{c}} \underline{0000-0002-1658-5539}$ \\ ${ }^{1}$ Instituto Nacional de Enfermedades Respiratorias Ismael Cosío Villegas, Ciudad de México, \\ México \\ ${ }^{2}$ Universidad Nacional Autónoma de México, Escuela Nacional de Enfermería y Obstetricia, \\ Ciudad de México, México
}

Recibido: 10 agosto 2019

Aceptado: 7 julio 2020

\section{RESUMEN}

Introducción: Actualmente el síndrome de agotamiento profesional y el cuidado perdido de enfermería (todo cuidado que se omite en parte o totalmente durante la atención) son problemas que afectan al profesional y al paciente disminuyendo la calidad y seguridad de los cuidados. El propósito de este artículo es analizar la evidencia de los informes que relacionan estos dos problemas y las soluciones propuestas.

Objetivo: Identificar la relación entre el síndrome de agotamiento profesional y el cuidado perdido de enfermería con base en la literatura consultada, para reconocer las causas en común de los dos problemas y establecer posibles soluciones.

*Autora para correspondencia. Correo electrónico: mariferrabadan@gmail.com 
Metodología: Revisión narrativa de la literatura, con una búsqueda realizada en marzo de 2019 en las bases de datos MEDLINE, LILACS, SCOPUS y SciELO, utilizando DeCS, MeSH y términos libres. Después de la lectura crítica de los documentos obtenidos, con base en los niveles de evidencia de la Joanna Briggs Institute y las guías CASPe, quedó una muestra de 14 artículos.

Resultados y conclusiones: Se encontraron distintos informes acerca de la relación del cuidado perdido con el síndrome de agotamiento profesional, algunos sitúan el síndrome como predisponente para el cuidado perdido, unos más el agotamiento como resultado de la omisión y otros como mediadores para la calidad y la seguridad de la atención. Los autores coinciden en que los factores para la aparición del agotamiento y el cuidado perdido son el ambiente laboral deficiente y las fallas en las relaciones interprofesionales.

Palabras clave: Agotamiento profesional; enfermería; cuidado perdido; cuidado dejado de lado; racionamiento implícito de la atención; cuidado sin terminar; México.

\section{ABSTRACT}

Introduction: Currently, the burnout syndrome and the nursing missed care (every care which is partially or totally omitted during nursing attention) are problems which have impacts on healthcare professionals and patients reducing the quality and safety of care. The purpose of this article is to analyze the evidence regarding the relationship between these two problems and the related proposed solutions.

Objective: To identify the relationship between the burnout syndrome and nursing missed care based on the consulted literature in order to identify common causes to both, and propose possible solutions.

Methodology: This a narrative review on the literature in the MEDLINE, LILACS, SCOPUS and SciELO using DeCS, MeSH, and free terms. The search was conducted in March, 2019. After the critical reading of the obtained documents, and based on the evidence levels proposed by the Joanna Briggs Institute, and on the CASPe guidelines, 14 articles were kept as the final sample.

Results and Conclusions: Diverse reports regarding the relationship between the burnout syndrome and nursing missed care were found. Some of these place the burnout syndrome as a predisposing factor to nursing missed care, others refer burnout as a result of omission, while others suggest both factors mediate in the quality and safety of healthcare. The authors agree that factors which contribute to both burnout or missing care are unfavorable working environments and deficient interprofessional.

Keywords: Burnout, professional; nursing; missed care; care left undone; implicit rationing of care; unfinished care; Mexico.

\section{RESUMO}

Introdução: Atualmente a síndrome de esgotamento profissional e o cuidado perdido de enfermagem (todos os cuidados que são parcial ou totalmente omitidos durante a atenção) são problemas que afetam o profissional e o paciente reduzindo a qualidade e segurança dos cuidados. O propósito deste artigo é analisar a evidência dos relatórios que relacionam estes dois problemas e as soluções propostas. 
Objetivo: Identificar a relação entre a síndrome de esgotamento profissional e a omissão de cuidados da enfermagem com base na literatura consultada, para reconhecer as causas em comum dos dois problemas e estabelecer possiveis soluções.

Metodologia: Revisão narrativa da literatura, com uma busca realizada em março de 2019 nas bases de dados MEDLINE, LILACS, SCOPUS e SciELO, utilizando DeCS, MeSH e termos livres. Depois da leitura crítica dos documentos obtidos, com base nos níveis de evidência da Joanna Briggs Institute e as guias CASPe, restou uma amostra de 14 artigos.

Resultados e conclusões: Encontraram-se distintos relatórios ao respeito da relação do cuidado perdido com a sindrome de esgotamento profissional, alguns situam a síndrome como fator predisponente para o cuidado perdido, alguns outros somados ao esgotamento como resultado da omissão e outros como mediadores para a qualidade e a segurança da atenção. Os autores concordam que os fatores para a aparição do esgotamento e o cuidado perdido são o ambiente laboral deficiente e as falhas nas relações interprofissionais.

Palavras-chave: Esgotamento profissional; enfermagem; cuidado perdido; cuidado deixados de lado; racionamento implícito da atenção; cuidado sem terminar; México.

\section{INTRODUCCIÓN}

Avedis Donabedian definió la calidad en salud como el tipo de atención que maximizará el bienestar del paciente, una vez teniendo en cuenta el balance de ganancias y pérdidas relacionadas con todas las partes del proceso de atención ${ }^{1}$. De igual forma, estableció el Modelo de Calidad de la Atención Médica, el cual estipula tres dimensiones: la estructura definida como los atributos de los sitios/ espacios, recursos materiales y humanos, y aspectos organizacionales que facilitan el cuidado; el proceso como todas aquellas acciones que se hacen para dar y recibir atención y el resultado como los efectos de la atención en el estado de salud de los pacientes y las poblaciones. Ninguna de las tres dimensiones puede permanecer aislada de la otra, y cada una repercutirá en el siguiente; por ende, si la estructura y/o el proceso tienen alguna falla, habrá un efecto negativo en los resultados.

En la dimensión del proceso se encuentra el cuidado perdido de enfermería, el cual está catalogado como un error de omisión, este tipo de errores son definidos como aquellos que se cometen cuando hay una falta de acción ${ }^{2}$. El cuidado perdido se considera una falla dentro del Modelo de Calidad de la Atención Médica ${ }^{1}$ que lleva a resultados negativos en el paciente.

Aiken et al. ${ }^{3}$ hablaron por primera vez de este problema en el International Hospital Outcomes Study bajo el término nursing care left undone. Posteriormente, Sochalski utilizó los términos task undone y unfinished care definiéndolo simplemente como tareas de enfermería que se dejaron de realizar porque no tenían tiempo para realizarlas 4.

En el 2006, Kalisch5se refirió al cuidado perdido con el término missed nursing care, definiéndolo como cualquier aspecto de la atención requerida del paciente que se omite (ya sea en parte o en su totalidad) o se retrasa y propuso el Missed Nursing Care Model, basándose en el modelo de Donabedian ${ }^{1}$ incluidas sus tres dimensiones, y el instrumento MISSCARE, el cual cuenta con una primera sección de 24 ítems para medir las actividades que más se pierden y una segunda sección con 17 ítems acerca de las razones por las cuales puede suceder el cuidado perdido ${ }^{6}$.

Un año después Schubert et al.7, utilizaron el término implicit rationing of nursing care definido como la retención o el incumplimiento de las medidas de enfermería necesarias para los pacientes 
debido a la falta de recursos, establecieron un modelo basado en el International Hospital Outcomes Study 8 el cual también contempla las tres dimensiones (estructura, proceso y resultados) y el instrumento Basel Extent of Rationing of Nursing Care (BERNCA) que cuenta con 20 ítems divididos en cuatro dimensiones: Actividades de la vida diaria; Cuidado y apoyo; Rehabilitación, instrucción y educación; Monitoreo y seguridad; y Documentación?

Finalmente en 2014 Ausserhofer et al..$^{10}$, acuñaron el término care left undone al que definieron como: actividades de enfermería que se omiten parcial o totalmente (se dejan de lado) cuando la escasez de recursos hace imposible la prestación de toda la atención necesaria.

El cuidado perdido de enfermería no solo ocasiona resultados negativos en el paciente, sino también produce sentimientos de culpa e insuficiencia en el profesional de enfermería, lo cual puede llevarlo a sufrir el Síndrome de Agotamiento Profesional o Burnout ${ }^{11}$. Este último se define como una forma inadecuada de afrontar el estrés emocional crónico cuyos rasgos principales son el agotamiento emocional, la despersonalización y la baja realización personal presentada en prosionales que trabajan en contacto directo con otras personas²; la forma de medirlo es con el instrumento Maslach Burnout Inventory (MBI), desarrollado por Maslach y Jackson ${ }^{12}$. Dicha evidencia apunta que el síndrome de agotamiento y el cuidado perdido están presentes en los profesionales de enfermería y sus causas son muy parecidas; sin embargo, sus conclusiones sobre estos dos problemas son heterogéneas ya que algunos autores proponen que el primero es el detonante del segundo ${ }^{13-15} \mathrm{y}$ otros opinan lo contrario, ${ }^{10,11,16}$ pero ambos repercuten en la seguridad y calidad de la atención ${ }^{17,18}$.

El presente artículo tiene como propósito analizar la evidencia presentada en los informes que consideran la relación entre estos dos problemas ya que se ha visto que el síndrome de agotamiento en los trabajadores produce mayor cantidad de errores ${ }^{19}$. Ahora bien, no es concluyente si el cuidado perdido es un factor predisponente para que el personal de enfermería desarrolle el síndrome o si el síndrome de agotamiento hace más susceptibles a estos profesionales de omitir cuidados. Por ello, el objetivo de esta revisión es: analizar la relación entre el síndrome de agotamiento profesional y el cuidado perdido de enfermería con base en la bibliografía consultada; además se pretende reconocer las causas en común de los dos problemas para establecer posibles soluciones; y así responder la siguiente pregunta: ¿El cuidado perdido de enfermería es una causante del síndrome de burnout o el agotamiento profesional es un posible responsable de la omisión del cuidado?

\section{METODOLOGÍA}

Este estudio corresponde a una revisión narrativa de la literatura realizada conforme a la metodología de Guirao-Goris² ${ }^{20}$. Se realizó una búsqueda en marzo de 2019 utilizando las bases de datos MEDLINE/ PubMed, LILACS, SCOPUS y SciELO, con los Descriptores en Ciencias de la Salud (DeCS) y los Medical Subject Headings (MeSH) burnout professional y nursing. Debido a que la palabra cuidado perdido carece de un descriptor, se utilizaron los siguientes términos libres: missed nursing care, care left undone, unfinished care y implicitly rationing of nursing care. Después de esto se elaboró una estrategia de búsqueda en inglés y español, se realizaron diversas combinaciones con los términos identificados y el operador booleano AND (Tabla 1).

Derivado de la búsqueda en las diferentes bases de datos se encontraron 201 artículos, 33 fueron elegidos por título y una vez leído el resumen se seleccionaron 16. De los 16 artículos seleccionados se revisó en cada uno el listado de referencias para elegir las que tuvieran relación con la pregunta de investigación, a partir de esto se recuperaron 15 estudios más, para un total de 31 
Tabla 1. Conceptos para la búsqueda

\begin{tabular}{|c|c|c|c|}
\hline Palabra clave & Términos alternativos & DeCS & MeSH \\
\hline $\begin{array}{l}\text { Síndrome } \\
\text { de desgaste } \\
\text { profesional }\end{array}$ & $\begin{array}{l}\text { Síndrome de burnout } \\
\text { Síndrome de agotamiento profesional } \\
\text { Síndrome de desgaste ocupacional } \\
\text { Burnout } \\
\text { Desgaste profesional } \\
\text { Estrés profesional }\end{array}$ & $\begin{array}{l}\text { Español: Agotamiento Profesional } \\
\text { Portugués: Esgotamento profesional }\end{array}$ & $\begin{array}{l}\text { Burnout, } \\
\text { Professional }\end{array}$ \\
\hline Enfermería & & $\begin{array}{l}\text { Español: Enfermería } \\
\text { Portugués: Enfermagem }\end{array}$ & Nursing \\
\hline Palabra clave & \multicolumn{3}{|c|}{ Términos alternativos } \\
\hline $\begin{array}{l}\text { Cuidado } \\
\text { perdido* }\end{array}$ & \multicolumn{3}{|c|}{$\begin{array}{l}\text { Español: Omisión del cuidado, Cuidado implícitamente racionado } \\
\text { Cuidado de enfermería dejado de lado, Racionamiento de los cuidados de enfermería, } \\
\text { Cuidados de enfermería inacabados } \\
\text { Inglés: Missed nursing care, Nursing care left undone, Rationing of nursing care } \\
\text { Implicitly rationed care, Unfinished nursing care }\end{array}$} \\
\hline
\end{tabular}

${ }^{*}$ No cuenta con términos DeCS/MeSH por lo que se utilizaron términos alternativos utilizados en otros artículos publicados

artículos, de los cuales se eligieron 14 para su estudio a fondo, pues cumplían con los siguientes criterios de inclusión:

1. Publicados en el periodo 2014-2019

2. En idioma inglés y español (se incluyó un artículo en alemán por cumplir con los demás criterios)

3. Que incluyeran tres de los términos (DeCS/MeSH/términos libres seleccionados o los sinónimos) en la totalidad del texto (Tabla 1)

4. Estudios enfocados al personal de enfermería

5. Estudios cualitativos o cuantitativos

De estos 14 artículos, diez son estudios de corte transversal, una revisión sistemática, una revisión de la literatura, un estudio cualitativo y un estudio mixto, se clasificaron entre el nivel 4 y 5 de evidencia según los criterios del Joanna Briggs Institute ${ }^{21 .}$

Después de la selección se procedió a la lectura crítica con ayuda de las Guías CASPe tanto para las revisiones ${ }^{22}$ como para los estudios cualitativos ${ }^{23}$. Conforme a los criterios establecidos se clasificó con calidad buena la revisión sistemática y muy buena la integrativa; los dos estudios cualitativos, correponden a calidad muy buena. En los estudios transversales se utilizó el instrumento propuesto por Ciaponni ${ }^{24}$ con el cual se calificó la calidad de seis artículos como muy buena, tres como buena y uno como regular; asimismo se realizó una tabla de evidencias con los principales hallazgos de cada artículo (Tabla 2).

\begin{tabular}{|c|c|c|}
\hline Artículo & Metodología & Discusión \\
\hline 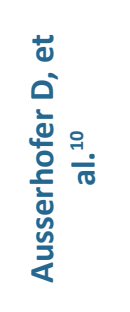 & $\begin{array}{l}\text { Estudio transversal, multinivel, multinacional, utilizó } \\
\text { los datos del RN4CAST en } 12 \text { países europeos (Bélgica, } \\
\text { Inglaterra, Finlandia, Alemania, Grecia, Irlanda, Polonia, } \\
\text { España, Suecia, Suiza, Holanda y Noruega), muestra de } \\
33659 \text { profesionales de enfermería de } 488 \text { hospitales. } \\
\text { El cuidado perdido se midió con } 13 \text { ítems, se utilizó la } \\
\text { encuesta PES-NWI para medir la carga de trabajo y las } \\
\text { tareas no enfermeras, se utilizaron dos ítems. Se realizó } \\
\text { análisis descriptivo y regresión lineal. }\end{array}$ & $\begin{array}{l}\text { Los hospitales con entornos laborales más } \\
\text { favorables tuvieron una menor prevalencia de } \\
\text { informes de cuidado dejado sin hacer. } \\
\text { La atención de enfermería que se deja sin hacer } \\
\text { crea potencialmente situaciones de conflicto moral } \\
\text { y de roles, por lo que puede influir en la satisfacción } \\
\text { laboral, la intención de irse y el agotamiento. }\end{array}$ \\
\hline
\end{tabular}




Estudio cualitativo derivado del análisis secundario de
datos de la encuesta en línea de la Association of Women's
Health Obstetric and Neonatal Nurses (AWHONN), una

m $\quad$ Estudio multinacional transversal RN4CAST, en 49

त) hospitales y una muestra de 1511 enfermeras(os). EI

1. racionamiento de la atención se midió con 13 ítems, se

ต midió el PES-NWI-R con un $\alpha$ de Cronbach entre $0.70 \mathrm{y}$ 0.89 . Se realizaron estadísticas descriptivas, un análisis de regresión, odds ratios, regresión logística en tres niveles y regresión separada.

Estudio transversal multicéntrico realizado en 162 casas de ancianos con una muestra de 3239 trabajadores del cuidado. Se utilizó el Care Worker Personnel Questionnaire, el BERNC-Nursing Home. Los problemas de salud física y el presentismo en 4 semanas antes de la encuesta se midió con un autoreporte, al igual que el cansancio y el insomnio. El agotamiento se midió con un solo ítem del $\mathrm{MBI}$. Se utilizaron dos subescalas del cuestionario PES-NWI. Se hicieron estadísticas descriptivas, modelos de regresión múltiple de la ecuación de estimación generalizada, modelos de regresión lineal para estimar los coeficientes $\beta$ y los intervalos de confianza, asimismo se calculó la d de Cohen.

Estudio transversal multicéntrico en 156 hogares de ancianos seleccionados aleatoriamente con una muestra de 4307 encuestados. Se utilizó el cuestionario BERNCA-

NH, el PES-NWI, el SAQ, el (HPSI). Se utilizaron estadísticas descriptivas, análisis de regresión múltiple, correlaciones intraclase, modelos multinivel, prueba de multicolinealidad con la varianza, factor de inflación, criterio de información de Akaike.

章

Revisión sistemática, se tomaron como fuentes Medline y CINAHL. Se buscaron artículos que incluyeran personal de enfermería de cualquier especialización que tuviera como propósito principal el establecimiento de prioridades, priorización o racionamiento de la atención y la inclusión de un aspecto ético en el establecimiento de prioridades. Se incluyeron 25 artículos. Se llevó a cabo análisis de datos inductivos y tablas descriptivas.

Estudio de corte transversal, multicéntrico en 155 residencias de ancianos, con una muestra de 4311 trabajadores. Se F. utilizó el instrumento PES-NWI, el SAQ con un $\alpha$ de 0.74 , el HPSI, el BERNCA, la calidad de atención, los estresores del trabajo, la falta de reconocimiento, carga de trabajo, falta de preparación. Para el análisis se utilizó un modelo de regresión logística de tres niveles para examinar la relación de dotación de personal, entorno laboral y racionamiento de la atención de enfermería con calidad de atención. Se utilizó criterio de información de Akaike para ajustes, siendo el valor más bajo un mejor ajuste.

Las consecuencias de falta de personal o personal inadecuado son: (a) atención no oportuna, incompleta u omisión completa (retrasada, sin terminar, o atención perdida); (b) aumento de la probabilidad de eventos adversos por falta de identificación, monitoreo y evaluación de los cambios o el deterioro de los pacientes; y (c) implicaciones profesionales negativas para las enfermeras.

Las actividades que más se pierden tienen que ver con atención psicosocial, mientras que las que menos se pierden son el manejo del dolor y la medicación oportuna. Los profesionales con agotamiento emocional presentan niveles más altos de racionamiento del cuidado. El principal factor que influye en que se racione menos el cuidado es la asignación adecuada de pacientes.

La salud mental, incluido el cansancio y el agotamiento emocional del profesional de enfermería, está relacionado con el racionamiento de las actividades tanto de la vida diaria como de la atención, la rehabilitación y el monitoreo. Las enfermedades, el dolor o el agotamiento relacionados con el trabajo pueden explicar las incongruencias comunes entre el conocimiento de los trabajadores sobre cómo llevar a cabo las tareas prescritas y su cumplimiento real de esas tareas de acuerdo con las pautas organizativas.

El estrés debido a la carga de trabajo, el personal de apoyo y la adecuación de los recursos tuvieron el mayor impacto en el racionamiento de la documentación y el estrés debido al conflicto. La falta de reconocimiento tuvo un impacto mayor en el racionamiento de la atención social. Tanto el racionamiento de actividades de la vida diaria como el cuidado, rehabilitación y monitoreo, fueron los más afectados por el estrés debido a la carga de trabajo. El estrés debido a la carga de trabajo, seguido de conflictos en el trabajo y la falta de reconocimiento tienen relación con el racionamiento del cuidado.

La priorización contribuye a la insatisfacción del paciente, reducción de los cuidados que brinda el profesional de enfermería, con ello al cuidado perdido y la pérdida de confianza al brindar cuidados. Además, genera conflicto de roles, dilemas éticos, tensión moral y disonancia emocional. Asimismo, sentimientos de culpa, insuficiencia, frustración e impotencia, lo que contribuye en el agotamiento, alta rotación y con ello, escasez de personal.

El entorno laboral, estrés laboral y racionamiento implícito de la atención de enfermería fueron factores importantes relacionados con la calidad de la atención. El clima de trabajo en equipo y la percepción inadecuada de los recursos de personal por parte de los trabajadores se relacionan con una menor calidad de la atención. La presencia combinada de diferentes condiciones de trabajo como un buen liderazgo, comunicación, trabajo en equipo y apreciación del personal, está relacionada con una mejor calidad de atención. 
Estudio transversal, muestreo aleatorio en 23 hospitales, con 1542 profesionales de enfermería que contestaron el cuestionario Pennsylvania Registered Nurse Questionnaire, $\stackrel{\text { - }}{\rightarrow-} \quad$ el PES-NWI, y el cuidado perdido se midió con 12 ítems con respuesta dicotómica. También se utilizó la subescala MBI-HSS y la seguridad del paciente se midió con la percepción de los profesionales y nueve eventos adversos considerados sensibles a la atención de enfermería. Se realizaron estadísticas descriptivas, análisis correlacional y estimaciones de confiabilidad, se implementó el SEM (Modelo de Ecuación Estructural).

\section{Revisión integrativa. Se utilizaron las bases de datos:} CINAHL, Medline, Embase databases, HINARI, Science Direct, Google y PubMed. Se obtuvieron 1336 artículos de los cuales se quedaron con 45 que incluyeran: un enfoque claro de enfermería, una descripción del concepto de calidad de atención en enfermería, una aclaración de los elementos utilizados por estos profesionales y pacientes para interpretar la calidad de la atención de enfermería.

․ Estudio descriptivo, exploratorio y transversal. Muestreo . aleatorio con 2387 enfermeras(os) del sector público y a privado de hospitales agudos y de ancianos. La encuesta tenía seis preguntas cuantitativas y una cualitativa para capturar las percepciones de la carga de trabajo. Los datos cuantitativos se analizaron con comparación de sectores, utilizando la prueba chi cuadrada y la prueba exacta de Fisher; los datos cualitativos se analizaron utilizando análisis temático.

Estudio transversal con una muestra aleatoria estratificada. Se utilizó el cuestionario de Aiken's IHOS y BERNCA en 3037 Registered Nurses en 51 hospitales. La calidad de la atención y la seguridad del paciente se midieron con dos ítems, el nivel de personal se midió con la cantidad de pacientes asignados en el último turno, las horas extra se calcularon con las horas reales trabajadas menos las horas programadas. Se realizaron análisis descriptivos, modelos de regresión logística multinivel y modelos de intercepción aleatoria.

Estudio transversal en 8 hospitales. Se seleccionaron 96 ¿ unidades con una muestra total de 1108 enfermeras(os).

a- Se utilizó el instrumento NWI-R, el MBI-HSS, se midió la 七 carga de trabajo, la libertad de decisión, el capital social, la satisfacción en el trabajo, la intención de dejar la profesión y la calidad de la atención. Se realizaron estadísticas descriptivas y coeficientes de correlación intraclase, modelado multinivel y modelos mixtos lineales de dos niveles con una intersección mixta.

Estudio multicéntrico, observacional, descriptivo y transversal en siete hospitales de España. Muestra estratificada de 635 enfermeras(os). Se utilizó un cuestionario autoadministrado con datos demográficos, educacionales, carga de trabajo, turno y el cuidado no completado RN4CAST. Se utilizó el cuestionario PES-NWI, el $\mathrm{MBI}$, el Horne and Östberg Morningness-Eveningness Scale, el Epworth Scale y el Pittsburgh Sleep Quality Index. Se utilizaron análisis descriptivos usando frecuencias relativas y absolutas, la media y desviaciones estándar, así como el análisis de $x^{2}$.
Un ambiente laboral favorable y la asignación adecuada de pacientes contribuyen a reducir el cuidado perdido; el agotamiento del profesional, mejora la seguridad del paciente y reduce el riesgo de presentar eventos adversos. La dedicación de más tiempo a tareas no profesionales se asoció a la atención de enfermería que se había dejado sin hacer y al agotamiento.
Existe relación entre la atención de enfermería de baja calidad con la omisión del cuidado y su mejora depende de tener suficiente personal competente (lo que a su vez permite que se dedique tiempo a los pacientes), y entornos laborales adecuados para que el personal de enfermería esté más satisfecho en su trabajo y experimente menos agotamiento.

Un gran número de profesionales de enfermería se sienten excluidos en la toma de decisiones, sintiéndose poco valorados e impotentes, esto influye en la satisfacción laboral y por lo tanto, en la retención de los profesionales. El no tener el tiempo suficiente para el paciente produce un agotamiento emocional que aumenta el estrés y el agotamiento en los profesionales, un factor que confunde la atención perdida.

Los cuidados que mayormente se pierden son la atención social y el confort. El aumento de la carga de trabajo, horas extraordinarias y entorno laboral inadecuado aumenta la percepción de la calidad de la atención como deficiente y aumenta las probabilidades de omitir el cuidado.

La participación en la toma de decisiones, un entorno de apoyo y reconocimiento, trabajo en equipo, liderazgo y comunicación en bucle cerrado son elementos que si fallan, es posible que se presente el cuidado perdido y el agotamiento.

Los niveles más altos de agotamiento se asociaron con resultados laborales desfavorables, quejas de pacientes y familiares, y abuso verbal de pacientes y familiares. Se confirmó el impacto de todas las dimensiones del entorno de práctica de los profesionales, en los resultados del trabajo y las variables de calidad de la atención, y también un impacto en los eventos adversos informados por enfermería.

La falta de tiempo del personal de enfermería no ayuda para: establecer relaciones interpersonales con el paciente, realizar educación sobre la salud, actualizar los planes de atención de enfermería y preparar al paciente y familia para el alta. La evidencia sugiere que las horas de sueño apropiadas y los días de descanso adecuados son factores protectores posibles, pero las proporciones más altas de número de pacientes por enfermera(o) se han relacionado con el agotamiento. 
Estudio transversal como parte del estudio RN4CAST en 12 países europeos con una muestra aleatoria de 31627 enfermeras(os) en 488 hospitales.

La encuesta se basó en el cuestionario validado del International Hospital Outcomes Study y el instrumento BERNCA. Se realizaron coeficientes de correlación intraclase a partir de modelos de intercepción aleatoria incondicional, modelos mixtos lineales generalizados binominales, distribuciones de Poisson y factor de inflación de varianza.
Trabajar horas extra en un turno, turnos de $\geq 12$ horas están asociados con informes de calidad reducida, independientemente del tiempo extra de trabajo y la duración de la semana laboral normal. El aumento de la fatiga, la pérdida del estado de alerta y el deterioro en la toma de decisiones son mecanismos plausibles para explicar la reducción de las calificaciones de calidad y seguridad con turnos más largos.

\section{RESULTADOS Y DISCUSIÓN}

La muestra consistió en 14 artículos analizados, dos revisiones, una sistemática, con un análisis de 24 artículos en inglés ${ }^{16} \mathrm{y}$ una integrativa con una revisión de 45 artículos también en inglés ${ }^{25}$, un estudio cualitativo realizado con una muestra de 844 miembros de enfermería obstétrica de Estados Unidos $^{11} \mathrm{y}$ un estudio mixto con una muestra de 2387 profesionales de enfermería de hospitales agudos de Australia, el cual contiene una parte cuantitativa (transversal) con seis preguntas y una parte cualitativa con una sola pregunta abierta ${ }^{26}$.

Los diez estudios restantes fueron de tipo cuantitativo con un diseño transversal y multicéntrico (tres de ellos multinacionales); siete estudios tienen una población de enfermería de hospitales agudos, dos de Asia ${ }^{18,27}$, cinco de Europa, ${ }^{10,13,28-30}$ y tres de residencias de adultos mayores de Europa ${ }^{14,15,17}$. El tamaño de las muestras oscila entre 635 y 33659 participantes.

Para medir el cuidado perdido cinco estudios utilizaron el instrumento Basel Extent of Rationing of Nursing Care (BERNCA) 14,15,17,27,30, otros dos 13 items del estudio RN4CAST ${ }^{11,13} \mathrm{y}$ uno más una encuesta de 12 ítems ${ }^{27}$, en cuanto al burnout y los factores estresantes cuatro utilizaron el instrumento Maslach Burnout Inventory (MBI) ${ }^{14,18,26,29}$ y dos el Health Professions Stress Inventory (HPSI)15, 17. Con un alfa de Cronbach en todos los instrumentos utilizados en los estudios entre 0.63 y 0.93.

En estos estudios se encontró el síndrome de burnout como un problema común en los servicios de salud. Dhaini et al..$^{14}$ reportaron un $37.7 \%$; Liu et al. ${ }^{18}$ un 40\%; Van Bogaert et al..$^{28}$ un $32.1 \%$; y Gómez et al. ${ }^{29}$ un $57.2 \%$ de agotamiento presente en personal de enfermería.

También se encontraron informes acerca de la cantidad de actividades omitidas por enfermería. Al respecto, Liu et al.$^{18}$ reportan un promedio de seis de doce tipos de tareas (50\%); Griffiths et al. ${ }^{30}$ un promedio de tres actividades de veinte (15\%); Zander et al..$^{13}$ un promedio de 5 actividades de trece (38.4\%) y Ausserhofer et al. ${ }^{10}$ un promedio de 3.6 actividades de trece (27.6\%). Sobre la proporción de este personal que omite actividades, Cho et al. ${ }^{27}$ indican un $82 \%$; Ausserhofer et al. ${ }^{10}$ un $27.6 \%$; Zander et al..$^{13}$ un $92.6 \%$ y Griffiths et al..$^{\circ}$ un $88 \%$. Referente a las actividades que más se omitieron, cinco autores reportan los porcentajes encontrados en sus estudios con valores entre $22.2 \%$ y $82 \%$ para confort/hablar con los pacientes; entre $17.2 \%$ y 54\% para enseñar/aconsejar a los pacientes y familiares; y entre $29.7 \%$ y 54\% para desarrollar/ actualizar los planes de atención. En los cuidados que menos se omiten está el manejo del dolor con un porcentaje entre $0.6 \%$ y 19\%; los tratamientos y procedimientos entre $1.1 \%$ y $19.4 \%$ y la administración de medicamentos en tiempo entre $5.5 \%$ y $21 \% \%^{10,13,15,27,29}$. Como podemos observar, los artículos son consistentes en los cuidados más omitidos, la mayoría corresponden a la atención psicosocial, la planificación y documentación de la atención; en cambio, las actividades enfocadas en la comodidad física y el bienestar son las que se omiten de manera menos frecuente $\mathrm{e}^{10,13-16,26}$. 
En el desarrollo del síndrome de burnout y del cuidado perdido influyen varios factores, los cuales se encuentran inmersos en los elementos del Missed nursing care model, en las dimensiones de la estructura y el proceso, este último dividido en nursing process y nursing internal process ${ }^{6,8}$.

Como parte de los elementos de la estructura se encontró que el ambiente laboral juega un papel muy importante en el cuidado perdido y el síndrome de burnout ${ }^{16-18,28,30}$. En este ambiente laboral varios autores han descrito la influencia de la carga de trabajo y la dotación de personal, al respecto Zúniga et al. ${ }^{15}$ identificaron que la carga de trabajo es la fuente más frecuente de estrés, asociándolo a su vez con un mayor impacto en el racionamiento de la documentación [ $\beta 0.289$ y $\beta-0.282, p<0.05]$ y de las actividades de la vida diaria [ $\beta$ 0.130 y $\beta$ 0.243, $p<0.05$ ]; mientras que Cho et al. ${ }^{27}$ mencionan que al aumentar un paciente por cada profesional, la probabilidad de omitir cuidados de enfermería durante su atención es mayor. [OR 1.03, IC95\% 1.01-1.05].

Esta carga de trabajo se ve acentuada por el incremento de las tareas no profesionales ${ }^{10,18,29}$ (definidas como las tareas que no requieren capacitación profesional de enfermería ${ }^{10}$ ), la falla sistémica de los empleadores para asignar y regular los recursos adecuados, la inadecuada combinación de habilidades propiciada por demasiado personal inexperto ${ }^{11,26}$ y la sustitución de personal registrado por personal no tan capacitado 25 .

Otros factores relacionados con un entorno laboral desfavorable son las horas extraordinarias y los turnos más largos. Griffiths et al..$^{\circ}$ documentan que todos los turnos $>8$ horas se asociaron con aumentos en la tasa de atención no realizada [ $p<0.05]$ y Cho et al. ${ }^{27}$ informan que cuando las enfermeras trabajan más horas las probabilidades de reportar atención dejada sin realizar fueron más altas [OR 1.86, IC95\% 1.48-2.35]. A su vez, el impacto de los turnos largos y las horas extraordinarias produce agotamiento, lo que ocasiona una pérdida del estado de alerta y deterioro en la toma de decisiones ${ }^{30}$.

El nursing process integra el proceso de atención de enfermería con sus cinco etapas y los factores que lo afectan como son las relaciones interdisciplinares entre los profesionales de la salud ${ }^{6,8}$. Estas relaciones deben de ser llevadas a cabo de forma respetuosa, con participación de todo el equipo de salud en la toma de decisiones, en un entorno de apoyo y reconocimiento basado en confianza mutua, liderazgo y comunicación en bucle cerrado, ya que si estás relaciones tienen fallas o tensiones, es más posible que se presente el cuidado perdido y el agotamiento10, ${ }^{15-18,25-28}$, Zúniga et al. ${ }^{15}$ encontraron que el estrés debido al conflicto y la falta de reconocimiento tuvo un impacto mayor en la omisión de la atención social [ $\beta 0.268, p<0.05]$.

En el nursing process internal se engloban los valores, creencias, hábitos y la toma de decisiones de cada profesional, pero también se han incluido los factores de salud física y mental de estos ${ }^{6,8}$. Dhaini et al ${ }^{14}$ reportaron cinco factores que predijeron el racionamiento de las actividades de la vida diaria: dolor en las articulaciones [ $\beta$ 0.04, IC95\% 0.001-0.07, $p<0.05$ ], cansancio [ $\beta$ 0.04; IC95\% 0.002-0.08, $p<0.05$ ], cefalea [ $\beta$ 0.04; IC95\% 0.01-0.08, $p<0.05$ ], agotamiento emocional [ $\beta$ 0.11; IC95\% 0.07-0.15, $p<0.05]$, y presentismo (definido como la práctica de asistir a trabajar a pesar de la enfermedad) [ $\beta 0.05$, IC95\% 0.004-0.09, $p<0.05]$. De igual forma, se evaluaron los factores que predijeron el racionamiento en las actividades de cuidado, rehabilitación y monitoreo, siendo las más importantes el dolor en las articulaciones [ $\beta$ 0.05, IC95\% 0.01-0.09, $p<0.05$ ], el cansancio [ $\beta$ 0.07, IC95\% 0.03-0.11, $p<0.05$ ], cefalea [ $\beta$ 0.04, IC95\% 0.001-0.08, $p<0.05$ ], y el agotamiento emocional [ $\beta$ 0.2, IC95\% 1.16-0.24, $p<0.05$ ].

Con base en estos resultados descritos, los factores que provocan el cuidado perdido y el síndrome de burnout son los mismos, en cuanto a la relación entre los dos hay quien asegura que el burnout desencadena el cuidado perdido. Al respecto, Dhaini et al. ${ }^{14}$ afirman que el agotamiento emocional es 
una de las razones para que se produzca la racionalización de las actividades de enfermería, Zander et al. ${ }^{13}$ informan que los cuidadores con un alto nivel de burnout tenían más riesgo de racionar las actividades [OR 1.60, $\mathrm{p}<0.05$ ] y Zúñiga et al. ${ }^{15}$ aseveran que niveles más bajos de estrés laboral se asociaron con un menor racionamiento de la atención [actividades de documentación: $\beta$ 0.289 y $\beta-0.282$ y actividades de la vida diaria: $\beta 0.130$ y $\beta 0.243, p<0.05]$.

Por otra parte, hay autores que aseveran que elcuidado perdido es un elemento predisponente para desarrollar el síndrome de burnout, Suhonen et al. ${ }^{16}$ declaran que los profesionales de enfermería que establecen prioridades en el cuidado experimentan sentimientos de culpa e insuficiencia, angustia moral, frustración e impotencia, lo que puede llevarlos a sufrir estrés y agotamiento. Simpson et al. ${ }^{11}$ enuncian que las implicaciones para enfermería asociadas con la atención que no se realiza son estrés, agotamiento y una cultura de seguridad deficiente; y Ausserhofer et al. ${ }^{10}$ sostuvieron que la atención de enfermería que se deja sin hacer provoca conflictos morales y de roles, lo que puede aumentar el agotamiento.

En contraste, Liu et al. ${ }^{18}$ correlacionan el agotamiento y la atención dejada sin hacer pero el efecto entre ellos no fue significativo [ $\beta 0.025$ p $>0.05$ ], igualmente encontraron una relación proporcional para el cuidado perdido y el burnout con los eventos adversos [burnout y eventos adversos: $\beta$-0.0150, cuidado perdido y eventos adversos: $\beta 0.073 p<0.05]$ y una relación inversamente proporcional con el nivel de seguridad del paciente [burnout y seguridad: $\beta$-0.0359, cuidado perdido y seguridad: $\beta-0.0132 p<0.05]^{18}$. Zúñiga et $a{ }^{17}$ informan que las probabilidades de una mejor calidad de atención aumentaron con un menor racionamiento del cuidado, la rehabilitación y el monitoreo [OR 0.34 CI95\% 0.24-0.49], y un menor racionamiento de la atención social [OR o.80 CI95\% 0.69-0,92], estipulando los dos autores que el cuidado perdido y el síndrome de burnout son mediadores entre el ambiente laboral y la calidad de la atención.

\section{CONCLUSIONES}

Dentro de esta revisión encontramos que las actividades de enfermería más racionadas son aquellas relacionadas con la atención psicosocial y la planificación y documentación de la atención, mientras que las actividades que reflejan la atención física y el control se dejan de realizar con menos frecuencia. Esto nos hace suponer que los profesionales utilizan un sistema jerárquico consciente o inconsciente para priorizar las actividades, en el cual anteponen las necesidades físicas de sus pacientes por encima de las necesidades sociales y emocionales.

La racionalización de las necesidades sociales se ha relacionado con una calidad de la atención mala, percibida por los pacientes y por el profesional, produciéndole a éste desafíos éticos, conflictos entre los valores personales y profesionales, estrés, insatisfacción con su trabajo y haciéndolo susceptible para presentar agotamiento, por tanto, se plantea que el cuidado perdido puede ocasionar que aparezca el síndrome burnout en el personal de enfermería.

No obstante, también se ha encontrado que el síndrome puede desencadenar el cuidado perdido ya que, si el profesional presenta despersonalización y cinismo, se olvida de la atención centrada en el paciente, pudiendo explicar así las incongruencias comunes entre el conocimiento sobre cómo llevar a cabo las tareas prescritas y el cumplimiento real de estas.

La falta de atención centrada en el paciente conlleva a una calidad de la atención y a una seguridad de la estancia hospitalaria deficiente, por lo cual algunos autores han relacionado el agotamiento y el cuidado perdido como mediadores para que existan resultados indeseables en el paciente. 
Asimismo, se llegó a la conclusión de que existen factores que influyen en el cuidado perdido y el síndrome de burnout, los cuales podemos situar en las dos dimensiones del Modelo del cuidado perdido de enfermería, la estructura y el proceso y a estos dos problemas analizados en la dimensión de resultados.

Los resultados muestran la utilidad del Modelo de cuidado perdido de enfermería, ya que los factores que influyen para que se manifieste el cuidado perdido y el síndrome de burnout corresponden a elementos considerados en las dimensiones de Estructura y Procesos.

A continuación (Tabla 3), se dan algunas recomendaciones basadas en los artículos que se consultaron para disminuir estos problemas:

En las recomendaciones que se plantean en los artículos se menciona que si se implementa la disminución de la carga de trabajo, pero no se mejora el ambiente laboral, el cuidado perdido no menguará, a su vez aseguran que si se interviene en el ambiente de trabajo se podrían atenuar los efectos de los niveles más bajos de personal y estimar de manera más eficiente la cantidad de personal adicional necesaria. Por ello, esto nos hace concluir que el factor en el que los gestores de la atención deben de poner mayor énfasis es el ambiente de trabajo.

Otro punto importante es que estas recomendaciones no sólo deben de ser llevadas a cabo por el gestor, sino en todos los eslabones de la organización de los centros de atención a la salud, ya que si alguno de ellos falla dará como consecuencia una cadena de defectos en el sistema ocasionando la presencia del cuidado perdido y el síndrome de burnout, y a su vez disminuye la calidad de la atención y la seguridad del paciente.

\section{Tabla 3. Recomendaciones}

1 Promover un ambiente de trabajo basado en el apoyo, la confianza mutua, el reconocimiento, la apreciación del personal, el liderazgo, el trabajo en equipo y la participación.

2 Mejorar la comunicación entre los profesionales de la salud, con la ayuda de la estrategia en bucle cerrado.

3 Creación de equipos de calidad.

4 Aumento de la dotación de personal, trabajadores con experiencia, sin sustituir puestos de enfermería profesional por personal auxiliar y asignar recursos humanos de apoyo adecuados.

5 Índice Enfermera/Paciente adecuado, siempre y cuando se tomen en cuenta las patologías y las necesidades del paciente.

6 Evitar horas laborales extraordinarias y turnos más largos.

7 Disminución de las tareas no profesionales.

8 Capacitación constante del personal en gestión del cuidado, habilidades necesarias para la atención del paciente y comunicación.

9 Llevar a cabo la atención centrada en el paciente.

10 Integración de las actividades que más se omiten a los planes de atención.

11 Implementar evaluaciones del desempeño organizacional.

12 Garantizar la disposición de recursos materiales.

13 Medir periódicamente el nivel de cuidado perdido y del síndrome de burnout.

14 Tomar en cuenta la salud física y emocional del personal de enfermería, así como la reducción del presentismo.

Integrar el cuidado perdido y el síndrome de burnout a los planes de estudio de todos los niveles de

15 educación en enfermería tales como: sus causas, consecuencias y las formas de manejarlo, así como cursos de ética para identificar y articular los valores y principios éticos que deben sustentar sus decisiones de priorización. 
Si bien el cuidado perdido y el síndrome de burnout siempre estarán presentes, pues nunca habrá suficientes recursos para satisfacer todas las necesidades de atención de la salud, es importante incrementar la investigación en diferentes poblaciones con diferentes sistemas de salud para así comparar los factores que lo producen y poder implementar acciones preventivas y correctivas, con el fin de reducir los riesgos en los pacientes y en los profesionales de enfermería.

\section{RESPONSABILIDADES ÉTICAS}

Protección de personas y animales: No aplica ya que esta es una investigación documental.

Financiamiento: Este artículo es producto del proyecto Cuidado perdido de enfermería, ambiente laboral y su relación con los eventos adversos reportados en hospitales de alta especialidad (PAPITT IN306419).

Conflicto de intereses: Los autores declaran no tener conflicto de intereses.

Agradecimiento: Agradecemos a la DGAPA UNAM por el financiamiento recibido.

\section{REFERENCIAS}

1. Donabedian A. The quality of care. How can it be assessed? JAMA. 1988; 260(12): 1743-8. https://ng.cl/7ajkz

2. Kalisch BJ, Landstrom G, Williams RA. Missed nursing care: errors of omission. Nurs Outlook. 2009; 57(1):3-9. https://doi.org/10.1016/j.outlook.2008.05.007

3. Aiken LH, Clarke SP, Sloane DM, Sochalski JA, Busse R, Clarke H, et al. Nurses' reports on hospital care in five countries. Health Aff (Millwood). 2001; 20(3): 43-53. https://doi.org/10.1377/hlthaff.20.3.43

4. Sochalski J. Is more better?: the relationship between nurse staffing and the quality of nursing care in hospitals. Med Care. 2004; 42(2 Suppl):II67-73.https://doi.org/10.1097/01.mlr.0000109127.76128.aa

5. Kalisch BJ. Missed nursing care: a qualitative study. J Nurs Care Qual. 2006; 21(4): 306-13. https://doi.org/10.1097/00001786-200610000-00006

6. Kalisch BJ, Landstrom GL, Hinshaw AS. Missed nursing care: a concept analysis. J Adv Nurs. 2009; 65(7): 1509-17. https://doi.org/10.1111/j.1365-2648.2009.05027.X

7. Schubert M, Glass TR, Clarke SP, Schaffert-Witvliet B, De Geest S. Validation of the basel extent of rationing of nursing care instrument. Nurs Res. 2007; 56(6): 416-24.

8. https://doi.org/10.1097/01.NNR.0000299853.52429.62

9. Aiken LH, Clarke SP, Sloane DM. Hospital staffing, organization, and quality of care: cross-national findings. Nurs Outlook 2002; 50(5):187-94. https://doi.org/10.1067/mno.2002.126696

10. Schubert M, Glass TR, Clarke SP, Aiken LH, Schaffert-Witvliet B, Sloane DM, et al. Rationing of nursing care and its relationship to patient outcomes the Swiss extension of the international hospital outcomes study. Int J Qual Health Care. 2008; 20(4): 227-37.https://doi.org/10.1093/intqhc/mzno17

11. Ausserhofer D, Zander B, Busse R, Schubert M, De Geest S, Rafferty AM, et al. Prevalence, patterns and predictors of nursing care left undone in European hospitals: results from the multicountry crosssectional RN4CAST study. BMJ Qual Saf. 2014; 23(2):126-35.https://doi.org/10.1136/bmjqs-2013-002318

12. Simpson KR, Lyndon A, Ruhl C. Consequences of inadequate staffing include missed care, potential failure to rescue, and job stress and dissatisfaction. J Obstet Gynecol Neonatal Nurs. 2016; 45(4): 481-9o. https://doi.org/10.1016/j.jogn.2016.02.011

13. Maslach C, Jackson SE. The measurement of experienced burnout. Journal of ocuppational behavior. 1981; 2(2): 99-113. https://doi.org/10.1002/job.4030020205 
14. Zander B, Dobler L, Bäumler M, Busse R. Implizite Rationierung von Pflegeleistungen in deutschen Akutkrankenhäusern - Ergebnisse der internationalen Pflegestudie RN4Cast. Berlin: Technische Universität Berlin; 2014. http://dx.doi.org/10.1055/s-0033-1364016

15. Dhaini SR, Zúñiga F, Ausserhofer D, Simon M, Kunz R, De Geest S, et al. Are nursing home care workers' health and presenteeism associated with implicit rationing of care? A cross-sectional multi-site study. Geriatr Nurs. 2016; 38(1):33-8. https://doi.org/10.1016/j.gerinurse.2016.07.003

16. Zuñiga F, Ausserhofer D, Hamers JPH, Engberg S, Simon M, Schwendimann R. The relationship of staffing and work environment with implicit rationing of nursing care in Swiss nursing homes - A cross-secctional study. Int J Nurs Stud. 2015; 52(9):1463-74. https://doi.org/10.1016/j.jjurstu.2015.05.005

17. Suhonen R, Stolt M, Habermann M, Hjaltadottir I, Vryonides S, Tonnessen S, et al. Ethical elements in priority setting in nursing care: A scoping review. Int J Nurs Stud. 2018; 88: 25-42.

https://doi.org/10.1016/j.ijnurstu.2018.08.006

18. Zúniga F, Ausserhofer D, Hamers JPH, Engberg S, Simon M, Schwedimann R. Are staffing, work environment, work stressors, and rationing of care related to care workers' perception of quality of care? A cross-sectional study. J Am Med Dir Assoc. 2015; 16(10): 860-6.

https://doi.org/10.1016/j.jamda.2015.04.012

19. Liu X, Zheng J, Liu K, Baggs JG, Liu J, Wu Y, et al. Hospital nursing organizational factors, nursing care left undone, and nurse burnout as predictors of patient safety: A structural equation modeling analysis. Int J Nurs Stud. 2018; 86: 82-9. https://doi.org/10.1016/j.ijnurstu.2018.05.005

20. Méndez-Venegas J. Estrés laboral o síndrome de “burnout”. Act Pediatr Mex. 2004; 25(5): 299-302. https://bit.ly/32fEePZ

21. Guirao-Goris JA, Olmedo-Salas A, Ferrer-Ferrandis E. El artículo de revisión. Revista Iberoamericana de Enfermería Comunitaria. 2008; 1-25. https://bit.ly/31suIsd

22. Aromataris E, Munn Z (Editors). JBI Manual for Evidence Synthesis. Australia: JBI; 2020. https://doi.org/10.46658/JBIMES-20-01

23. Cabello JB. Plantilla para ayudarte a entender una revisión sistemática. En: CASPe. Guías CASPe de lectura crítica de la literatura médica. Alicante: CASPe; 2005.

24. Cano-Arana A, González-Gil T, Cabello-López JB. Plantilla para ayudarte a entender un estudio cualitativo. En: CASPe. Guías CASPe de lectura crítica de la literatura médica. Alicante: CASPe; 2010.

25. Ciaponni A. Artículo especial: Guía de lectura crítica de estudios observacionales en epidemiología (primera parte). Evid. Actuali. práct. ambul. 2010; 13(1): 135-40. https://n9.cl/m7t74

26. Koy V, Ynibhand J, Angsuroch Y, Fisher ML. Relationship between nursing care quality, nurse staffing, nurse job satisfaction, nurse practice environment, and burnout: literature review. Int J Res Med Sci. 2015; 3(8): 1825-31. http://dx.doi.org/10.18203/2320-6012.ijrms20150288

27. Hegney DG, Rees CS, Osseiran-Moisson R, Breen L, Eley R, Windsor C, et al. Perceptions of nursing workloads and contributing factors, and their impact on implicit care rationing: A Queensland, Australia study. J Nurs Manag. 2019; 27(2): 371-80. https://doi.org/10.1111/jonm.12693

28. Cho E, Lee NJ, Kim EY, Kim S, Lee K, Park KO, Sung YH. Nurse staffing level and overtime associated with patient safety, quality of care, and care left undone in hospitals: A cross-sectional study. Int J Nurs Stud. 2016; 60: 263-71. https://doi.org/10.1016/j.jjnurstu.2016.05.009

29. Van Bogaert P, Timmermans O, Weeks SM, Van Heusden D, Wouters K, Franck E. Nursing unit teams matter: Impact of unit-level nurse practice environment, nurse work characteristics, and burnout on nurse reported job outcomes, and quality of care, and patient adverse events- a cross-sectional survey. Int J Nurs Stud. 2014; 51(8): 1123-34. https://doi.org/10.1016/j.jpurstu.2013.12.009 
30. Gómez-García T, Ruzafa-Martínez M, Fuentelsaz-Gallego C, Madrid JA, Rol MA, Martínez-Madrid MJ, et al. Nurses' sleep quality, work environment and quality of care in the Spanish National Health System: observational study among different shifts. BMJ Open. 2016; 6(8): 1-11. https://doi.org/10.1136/bmjopen-2016-012073

31. Griffiths P, Dall' Ora C, Simon M, Ball J, Lindqvist R, Rafferty AM, et. al. Nurses' shift length and overtime working in 12 European countries. Med Care. 2014; 52(11): 975-81.

https://doi.org/10.1097/MLR.0000000000000233 